\title{
Creation of a Digital Passport for an Electronic Product and Generation of Design Solutions Based on It
}

\author{
Julia V. Donetskaya ${ }^{1}$ \\ ITMO University, Russia
}

\begin{abstract}
The work presents the activity analysis of enterprises in the instrumentation industry. This result shown the content of the digital passport is described in the form of a set of models, which makes it possible to use it at the stages of the life cycle of a product for the generation of design solutions. The work lists the variants of design solutions, as well as the tasks of their generation based on the analysis of parameters set by fuzzy variables. The required solution contains the associated components of the digital passport that are a part of the set specified by the values of the variables. The results obtained can be used to create a digital passport on the enterprises in the instrument-making industry.
\end{abstract}

Keywords. digital passport, generation of design solutions, variants of design solutions, tasks of generation design solutions.

\section{Introduction}

A modern electronic product is a complex hardware and software system, the development and delivery of which is performed on the basis of customer requests. Its content determines the lists and terms of work, as well as their performers from among the divisions of the enterprise and various organizations. This predesignates the task of organizing their operational interaction - the exchange of relevant data for making design decisions. Its solution is divided into two stages.

At the first stage, enterprises put into operation and integrate such systems as Product Data Management (PDM), Enterprise Resource Planning (ERP), Manufacturing Execution System (MES) and / or Quality Management System (QMS), as well as electronic document management (EDMS), which makes it possible to increase the efficiency of interaction between departments [1,2]. As a result, at each stage of the life cycle, data about the product and the corresponding design and production procedures are formed, which form a digital passport of this product. At the second stage, the tasks of data exchange between different organizations are being solved.

Traditionally, the exchange is associated with correspondence by e-mail or with the transfer of technical documentation in hard copy. This leads to the emergence of human factor problems that affect the efficiency of making design decisions. In order to eliminate them, digital passport technologies are used, which means the introduction and

1 Corresponding Author: Donetskaya Julia V., ITMO University, Faculty of Secure Information Technology, 191002, Saint-Petersburg, ul. Lomonosova 9, lit.M, Russia; E-mail: donetskaya_julia@mail.ru 
synchronization of versions of the same systems, and models of stored data by all participants in the interaction, coordination of the formation rules and structure of a neutral data exchange format in the STEP language.

The defects identified in this case are obvious and more significant in the vector of development of Industry 4.0 [3-7]. This indicates the need to generalize data about the product and design-production procedures at all stages of the life cycle in the instrumentmaking industry, as well as the implementation of procedures for their analysis for making design decisions by enterprises.

Thus, the creation of a digital passport for an electronic product and the generation of design solutions based on it is a priority task, the results of which are presented in this article. This contains:

1. The activity analysis of enterprises in the instrument-making industry.

2. The variants of design solutions generated on the basis of a digital passport.

3. The tasks of generation of design solutions based on a digital passport.

\section{Activity analysis of enterprises in the instrument-making industry}

The activity of an instrument-making enterprise is performed at the stages of life cycle of an electronic product, each of which is characterized by different design and production procedures. However, for research, only those that make it possible to form and / or change data about an electronic product, which are represented by the corresponding information objects are determined with their substantive and requisite parts.

The stage of concluding an agreement implies the implementation of such procedures as: consideration and approval of applications for the development or supply of an electronic products; preparation, registration and signing of an agreement with a customer; registration and signing of an agreement with a co-contractor.

As a result, linked documents are created. These include, first of all, an application for the development or supply of products with a corresponding agreement with the customer, and when involving third-party organizations (there may be several of them) - agreements with co-executors. This allows to perform the following development phase procedures:

1. Development, coordination and approval of the work schedules.

2. Management of updating data on standard and other products (purchased component parts - PCP), materials.

3. Development and modification of design data [2].

4. Coordination, approval and submission of documentation to the archive.

Thus, a set of design data about the product is created in the form of the electronic structure of the product in the PDM-system (ESI-PDM), containing documentation, as well as 3D models of parts and assembly units [8]. The results obtained are used in the preparation of production:

1. Development, coordination and approval of the production schedules.

2. Development and modification of manufacturing data.

3. Coordination, approval and submission of documentation to the archive.

4. Recording comments and decisions.

5. Preparation and registration of the agreement for the supply. 
6. Recording and registration of the lot entry of supply products.

7. Formation of a production plan for the manufacture of products.

These procedures make it possible to create, on the basis of design data, a set of corresponding manufacturing data about a product, as well as to purchase PCP, materials and products of co-contractors. The information obtained is used at the production stage when performing the following procedures:

1. Verification of purchased products and materials upon delivery from the warehouse.

2. Assignment of identification numbers to parts and assembly units (PAU).

3. Fixing the composition of manufactured assembly units.

4. $\quad$ Fixing the execution of PAU manufacturing operations.

5. Recording comments and decisions during the production of PAU products.

As a result, the structure of a product sample (SPS) is formed, the elements of which are PAU copies, manufactured at the enterprise and purchased under agreements [9]. In the production process, for each of them, the values of quality parameters are monitored and the compliance of the PAU with the developed documentation is being checked.

After the completion of the product production, cycles of its tests are performed, and their successful completion means the possibility of moving to the stages of operation and repair. This means performing the following procedures: registration of shipment of the finished products sample from the warehouse; registration of the complaint report and the result of the refusal study; registration and control of the service request.

At the same time, for a given SPS element, a failure in operation and the result of its study is recorded, for an SPS, the product is shipped to the customer, as well as the serial number of the component parts of the product from the set of spare parts and accessories when processing a service request.

Thus, the stages of the life cycle are set in accordance with design and production procedures $\mathrm{Pr}=\left(p r_{1}, p r_{2}, \ldots, p r_{n}\right)$, during the implementation of which data about an electronic product is created $D=\left(d_{1}, d_{2}, \ldots, d_{k}\right)$ in the form of information objects of ERP, PDM, MES, QMS systems or EDMS.

The values of each of them allowed to describe the components of the digital passport

$$
R(C)=\left(\begin{array}{cccc}
c_{11} & c_{12} & \ldots & c_{1 n_{1}} \\
c_{21} & c_{22} & \ldots & c_{2 n_{2}} \\
\ldots & \ldots & c_{i j} & \ldots \\
c_{k 1} & c_{k 2} & \ldots & c_{k n_{k}}
\end{array}\right)=\left(\begin{array}{c}
C_{1} \\
C_{2} \\
\ldots \\
C_{k}
\end{array}\right),
$$

where $c_{i j}=\left\langle d_{i}, p r_{j}\right\rangle, i=\overline{1, k}, j=\overline{1, n_{k}} ; n_{k} \leq n-$ an element of a digital passport. Moreover $c_{i j}=1$, if the given object $d_{i} \in D$ formed as a result of a specific procedure $p r_{j} \in \operatorname{Pr}$ и $c_{i j}=0$ otherwise.

The results obtained also allowed the creation of many parameters of the components of the digital passport $U=\left(u_{1}, u_{2}, \ldots, u_{P}\right)$ which are used in a model for describing 
components

$$
G(C)=\left(\begin{array}{cccc}
g_{11}\left(C_{1}\right) & g_{12}\left(C_{1}\right) & \ldots & g_{1 P}\left(C_{1}\right) \\
g_{21}\left(C_{2}\right) & g_{22}\left(C_{2}\right) & \ldots & g_{2 P}\left(C_{2}\right) \\
\ldots & \ldots & g_{i j}\left(C_{i}\right) & \ldots \\
g_{k 1}\left(C_{k}\right) & g_{k 2}\left(C_{k}\right) & \ldots & g_{k P}\left(C_{k}\right)
\end{array}\right)=\left(\begin{array}{c}
G_{1}\left(C_{1}\right) \\
G_{2}\left(C_{2}\right) \\
\ldots \\
G_{k}\left(C_{k}\right)
\end{array}\right),
$$

where each element $g_{i j}\left(C_{i}\right) \in G_{i}\left(C_{i}\right), i=\overline{1, k}, j=\overline{1, P}$ is described by parameters and formed at a specific stage of the life cycle.

Components are linked

$$
X(C)=\left(\begin{array}{cccc}
0 & X_{12}\left(C_{1}, C_{2}\right) & \ldots & X_{1 k}\left(C_{1}, C_{k}\right) \\
X_{21}\left(C_{2}, C_{1}\right) & 0 & \ldots & X_{2 k}\left(C_{2}, C_{k}\right) \\
\ldots & \ldots & X_{i j}\left(C_{i}, C_{j}\right) & \ldots \\
X_{k 1}\left(C_{k}, C_{1}\right) & X_{k 2}\left(C_{k}, C_{2}\right) & \ldots & 0
\end{array}\right),
$$

where

$$
X_{i j}\left(C_{i}, C_{j}\right)=\left\{\begin{array}{ll}
0, & \operatorname{out}\left(G_{i}\left(C_{i}\right)\right) \neq \operatorname{in}\left(G_{j}\left(C_{j}\right)\right) \\
1, & \operatorname{out}\left(G_{i}\left(C_{i}\right)\right)=\operatorname{in}\left(G_{j}\left(C_{j}\right)\right)
\end{array} .\right.
$$

Then, on the basis of the above, it is logical to assert about the possibility of using the content of a digital passport for the generation of design solutions.

\section{Variants of design solutions generated on the basis of a digital passport}

So, when considering and approving an application for the development or supply of products, design solutions are formed in the form of lists of products and their component parts to be developed (including products of co-contractors and its suppliers) and lists of documentation for component parts of the product to be adjusted.

The procedures for developing, reconciliation and approving the work schedules use design solutions in the form of lists of developed and changed component parts of the product and products of co-contractors and / or documentation developed or adjusted for component parts of the product, as well as the results of fulfilling the positions of the operational work schedule.

To manage the update of PCP data, a design solution is required that contains a list of approved PCPs, products of co-contractors and materials.

Development and modification of design data requires information to create tabular design documentation and ESI. Similar design solutions are required for the development and modification of software for the device and a set of operational documentation - data for creating tabular and operational documentation, as well as ESI. 
When performing the procedure for the development, coordination and approval of the production schedule, information about the component parts of the product to be produced is used; information about PCPs, materials, products of co-contractors and their suppliers; including the results of fulfilling the positions of the application for the start of production.

The development and modification of manufacturing data is performed according to the data for the creation of technological documentation for the manufacture of the product and the electronic structure of the product.

To prepare and register an agreement for the supply, data on the PCP, materials, products of co-contractors and their suppliers are generated.

The formation of a production plan for the manufacture of products requires data on the constituent parts of the product to be produced, the current production load and the results of the production operations.

When registering a complaint act and the result of a failure study, the lists of documentation for the component parts of the product subject to correction, lists of PCPs, materials, products of co-contractors and their suppliers, as well as lists of data on the component parts of the product subject to production are taken into account.

Registration and control of the service request requires the formation of data on the changeable component parts of the product and the corrected documentation, the component parts related to spare parts and the results of fulfilling the plan items for processing the service request.

The listed options for design solutions can be grouped and get the following list of tasks: the task of searching for data about products and their components; the task of finding data about PCPs, materials, products of co-contractors and their suppliers; the task of finding data for the development and correction of documentation; the task of finding data on the documentation for the component parts of the product; the task of monitoring the fulfillment of the positions of the unified task; the task of managing production operations.

\section{Tasks of generation of design decisions based on digital passport}

The solution to any of these problems is a set of related components of a digital passport, which means the formation of matrix elements (2) that satisfy condition (3) in accordance with the values of the analyzed parameters $P R$. Moreover, the lists of parameters are defined for each task in the form of fuzzy variables:

$$
\langle P, A, \mu(P)\rangle
$$

where $P$ - the name of the parameter from the list below; $A$-parameter value; $\mu(P)$ - parameter membership function.

For example, for the task of searching for data about a product and their constituent parts are defined: type of product and component, manufacturer of the product and component, sign of a component part of the product, the presence of comments on the documentation, a sign of products of inadequate quality.

For the task of searching for data on documentation for component parts of a product are defined: type of product component; sign of a component part of the product; document sign; the presence of comments on the documentation. 
For the task of relating to the control of the execution of the positions of the unified task are defined: type of work; monitored state.

To solve the problem of managing production operations are defined: the presence of comments on the documentation; availability of products in stock; sign of the production process; sign of products of inadequate quality.

Then, the generation of design solutions based on digital passport, it is necessary to solve a number of problems associated with the analysis and selection of descriptions of the components of a digital passport. The first task based on the calculation of the values of the membership functions of fuzzy variables and the second task based on the TakagiSugeno-Kanga algorithm.

This will allow developing a similarity criterion given by the least squares method

$$
\Delta(\beta)=\sum_{i=1}^{K}\left(G_{i}\left(\overrightarrow{C_{i}}\right)-G_{i}^{*}\left(\overrightarrow{C_{i}}\right)\right)^{2}=(G-\beta \cdot X)^{T} \cdot(G-\beta \cdot X) \rightarrow \min ,
$$

where

elements $G_{i}\left(\vec{C}_{i}\right) \in G(C)(i=\overline{1, K} ; K \leq k)$ (1) are calculated using the theory of fuzzy sets and elements of discrete mathematics; elements $G_{i}^{*}\left(\overrightarrow{C_{i}}\right)=a \cdot \sum_{j=1}^{P} b_{j} \cdot R P_{j} \cdot g_{i j}\left(\overrightarrow{C_{i}}\right)$ are calculated using the theory of pattern recognition and the theory of decision making;

$\beta=\left(a, b_{1}, b_{2}, \ldots, b_{K}\right)-$ column vector;

$X=R P \cdot G-$ deterministic matrix, dimension $K \times P$.

Consequently, the search for design solutions that satisfy the similarity criterion means calculation the elements of the column vector, according to an expression of the form:

$$
\beta=\left(X^{T} \cdot X\right)^{-1} \cdot\left(X^{T} \cdot G\right)
$$

The generated design solution is one of the lists in the form of the values of the digital passport components. For the task of searching for data about products and their components: designation of the component part of the product; name of the component part of the product; name of the manufacturer of the product component; sign of referring a component part of the product to spare parts; sign of a component part of the product.

For the task of finding data for the development and correction of documentation: designation of the component parts of the product; designation of documentation; the name of the component parts of the product; title of the documentation; documentation format; version of the documentation; the number of component parts of the product included in the assembly unit.

For the task of searching for data on documentation for component parts of the product: designation of documentation; title of the documentation; version of the documentation; the state of the version of the component parts of the product; state of the documentation version. 
For the task of monitoring the fulfillment of the positions of the unified task: number of the unified task; position number of the unified task; name of the position of the unified task; result of execution of the task position and status of the result of the task position execution.

For the task of managing production operations: the number of the production operation; name of the production operation; designation of the manufactured product copy and the result of the production operation.

The set of conditions formed in this way determines the area, falling into which characterizes the desired design solution.

\section{Conclusion}

Thus, it is shown: the content of the digital passport is determined by the components; the set of components allows you to form the content of the digital passport and use it to generate different design solutions; the tasks of generation design solutions based on digital passport are formulated.

The results obtained can be used to create a digital passport on the enterprises in the instrument-making industry.

\section{References}

[1] Donetskaya J.V., Kuznetsova O.V., Tushkanov E.V., Kuznetsov A.Y. Formation and analysis of engineering alternatives for an integrated electronic product description system. In: Control in Technical Systems (CTS), 2017 IEEE II International Conference on - 2017; 2017. pp. 397-400.

[2] Donetskaya J.V., Kuznetsova O.V., Tushkanov E.V., Kuznetsov A.Y, Gatchin Yu.A. Mathematical model of a digital passport for an electronic product. In: International Conference on Soft Computing and Measurements - 2018, vol. 1. 2018. pp. 438-441.

[3] Wagner T., Herrmann Ch., Thiede S. Industry 4.0 impacts on lean production systems. Procedia CIRP 63. 2017. pp. 125-131.

[4] Chen Y. Integrated and intelligent manufacturing: perspectives and enablers. Engineering 3. 2017. pp. 588595.

[5] Doshi A., Smith R.S., Thomas B.H., Bouras C. Use of projector based augmented reality to improve manual spot-welding precision and accuracy for automotive manufacturing. The International Journal of Advanced Manufacturing Technology 89. 2017. pp. 1279-1293.

[6] Uva A.E., Gattullo M., Manghisi V.M., Spagnulo D., Cascella G.L., Fiorentino M. Evaluating the effectiveness of spatial augmented reality in smart manufacturing a solution for manual working stations. The International Journal of Advanced Manufacturing Technology 94. 2018. pp. 509-521.

[7] Abramovichi M., Gobel J. Ch., Savarino Ph., Gebus Ph. Towards smart product lifecycle management with an integrated reconfiguration management. International Federation for Information Processing. 2017. pp. 489-498.

[8] Osepyan A.K. The state and prospects for the development of engineering data management systems in the branch of FSUE TsENKI - Scientific Research Institute of PM named after Academician V.I. Kuznetsov. In: Proceedings of the XVII conference of young scientists "Navigation and traffic control". SPb.: State Scientific Center of the Russian Federation "Concern" TsNII "Elektropribor". 2015. pp. 168-174.

[9] Shakhovtsev E.V., Balandin A.I., Shvidko A.E. Interaction of automated production preparation systems and product life cycle management: promising options. In: Proceedings of the conference "Information technologies in management (ITU-2014)". 2014. pp. 431-439. 